\title{
Body Composition, Nutritional Status and Mortality in Peritoneal Dialysis Patients
}

Morrell M. Avram*, Paul A. Fein, Samuel Agahiu, William Hartman, and Jyotiprakas Chattopadhyay

Avram Division of Nephrology, S.U.N.Y. Downstate Medical Center U.H.B. at Long Island College Hospital, Brooklyn, New York, USA

\begin{abstract}
Background: Protein energy malnutrition is highly prevalent and a risk factor for mortality in peritoneal dialysis (PD) patients. In this study we have investigated the association of body composition parameters with nutritional status and outcomes in PD patients.

Methods: We enrolled 63 PD patients between November 2000 to May 2008. On enrollment, demographic, clinical and biochemical data were recorded. Patients were followed to May 2011. Body composition was determined by bioelectrical impedance analysis (BIA).

Results: Mean age was 54 years. At enrollment, the mean $( \pm \mathrm{SD})$ serum albumin, creatinine and $\mathrm{nPCR}$ were 3.71 $\pm 0.59 \mathrm{~g} / \mathrm{dL}, 11.38 \pm 4.2 \mathrm{mg} / \mathrm{dL}$ and $0.94 \pm 0.27 \mathrm{~g} / \mathrm{kg} /$ day respectively. Mean $( \pm \mathrm{SD})$ body mass index (BMI), phase angle (PA) and extracellular mass/body cell mass (ECM/BCM) ratio were $25.37 \pm 5.46 \mathrm{Lbs}_{\text {/inch }}^{2}, 6.06 \pm 1.6$ degrees and $1.21 \pm 0.2$ respectively. Albumin, and creatinine directly correlated with $B M I(p=0.027$ for albumin; $p<0.0001$ for creatinine) and PA ( $<<0.0001$ for albumin; $p=0.031$ for creatinine) and inversely correlated with $E C M / B C M$ ratio $(p=0.001$ for albumin; $p=0.07$ for creatinine). During the study period 26 patients expired. Patients who survived had higher BMI $(p=0.05)$, PA $(p=0.017)$ and lower ECM/BCM ratio $(p=0.04)$ compared to those who expired. In Cox's multivariate analysis, adjusting for confounding variables, higher levels of PA (Relative risk: $0.52, p=0.009$ ) and lower levels of $E C M / B C M$ ratio (Relative risk: $1.039, p=0.035$ ) were significantly and independently associated with increased survival in PD patients followed up to 11 years.
\end{abstract}

Conclusions: Estimates of BMI, PA and ECM/BCM ratio reflect nutritional status and are important predictors of long term survival in PD patients. It may be useful to incorporate these parameters in the management of malnutrition and overall health in these patients.

Keywords: Peritoneal dialysis; Bioimpedance analysis; Nutrition; Body composition; Nutritional markers; Mortality

Abbreviations: PD: Peritoneal Dialysis; BIA: Bioimpedance Analysis; BMI: Bmass Index; ECM: Extracellular Mass; BCM: Body Cell Mass; nPCR: normal Protein Catabolic Rate; PA: Phase Angle

\section{Introduction}

Although the survival rate of end-stage renal disease (ESRD) patients continues to improve, it remains markedly reduced compared to the general population. Adjusted rates for all-cause mortality are seven times greater for dialysis patients than for individuals in the general population [1]. Despite the increasing knowledge and improvement in dialysis treatment, mortality of PD patients remains high. Identification of various risk factors and aggressive risk factor modification are important strategies to improve outcomes in these patients.

Malnutrition is highly prevalent and is an important factor contributing to high morbidity and mortality in PD patients [2-4]. Lower levels of serum nutritional markers are associated with higher mortality in PD patients [5-8]. Low dietary protein intake and protein energy wasting have been reported to be associated with increased mortality in PD patients $[9,10]$.

BIA has been recognized as a simple, fast and noninvasive method for assessing the body composition and fluid status in PD patients [11]. We and others have investigated the relationship of BIA derived body composition parameters with nutritional markers, morbidity and mortality in PD patients $[12,13]$. We have reported that BIA derived $\mathrm{ECM} / \mathrm{BCM}$ ratio, a marker of malnutrition, is an independent predictor of survival in PD patients followed to 8 years [14]. In this study we have extended the follow-up period to 10 years and in addition to
$\mathrm{ECM} / \mathrm{BCM}$ ratio, we have investigated the relationships of two other important body composition parameters such as BMI and PA with nutritional markers and survival in PD patients. In the present study, we have analyzed the data in a different way to add new information to the literature.

\section{Materials and Methods}

We enrolled 63 PD patients between November 2000 to May 2008. On enrollment, demographic, clinical and biochemical data were recorded. BIA measurements were conducted using an impedance plethysmograph $(800 \mathrm{~mA}$ and $50 \mathrm{kHz})$. Patients' electrical impedance values, resistance and reactance were used for computerized calculation of the body composition parameters using Cyprus version 1.0 (BIA101; RJL/Akern Systems). Patients were followed until September, 2011. Long Island College Hospital Institutional Review Board approved this study protocol and informed consent was obtained from each patient.

\section{Statistical analysis}

Continuous variables are expressed as mean \pm SD. For selected

*Corresponding author: Morrell M. Avram, MD, Avram Division of Nephrology S.U.N.Y. Downstate Medical Center U.H.B. at Long Island College Hospital, 339 Hicks Street, Brooklyn, New York 11201, USA, Tel: (718) 780-1247; Fax: (718) 7801415; E-mail: morrell.avram@downstate.edu

Received January 31, 2012; Accepted April 09, 2012; Published April 11, 2012

Citation: Avram MM, Fein PA, Agahiu S, Hartman W, Chattopadhyay J (2012) Body Composition, Nutritional Status and Mortality in Peritoneal Dialysis Patients. J Nephrol Therapeutic S10:003. doi:10.4172/2161-0959.S10-003

Copyright: (c) 2012 Avram MM, et al. This is an open-access article distributed under the terms of the Creative Commons Attribution License, which permits unrestricted use, distribution, and reproduction in any medium, provided the original author and source are credited. 
comparisons between group means, parametric (t-test) or nonparametric (Mann-Whitney test) tests were used. Correlations were reported as either the Pearson correlation coefficient or the Spearman rank correlation coefficient. Observed survival was computed by the Kaplan-Meier method [15]. Independent predictors of survival were determined by Cox regression analysis. Calculations were performed using SPSS for Windows 12.0.1 (IBM Corporation, Armonk, New York).

\section{Results}

\section{Demographics and biochemical characteristics}

Mean age was $54 \pm 16(\mathrm{SD})$ years. Fifty-five percent were women, sixty-three percent were of African descent and twenty-five percent were diabetic. At enrollment, the mean $( \pm$ SD) serum albumin, creatinine and $\mathrm{nPCR}$ were $3.71 \pm 0.59 \mathrm{~g} / \mathrm{dL}, 11.38 \pm 4.2 \mathrm{mg} / \mathrm{dL}$ and $0.944 \pm 0.27 \mathrm{~g} / \mathrm{kg} /$ day respectively. Mean $( \pm \mathrm{SD})$ body mass index (BMI), phase angle (PA) and extracellular mass/body cell mass (ECM/ $\mathrm{BCM})$ ratio were $25.37 \pm 5.46 \mathrm{Lbs} / \mathrm{inch}^{2}, 6.06 \pm 1.6$ degrees and $1.21 \pm$ 0.2 respectively.

\section{Correlation of body composition parameters with serum nutritional markers}

Albumin, and creatinine directly correlated with BMI $(r=0.28$, $\mathrm{p}=0.027$ for albumin; $\mathrm{r}=0.48, \mathrm{p}<0.0001$ for creatinine $)$ and $\mathrm{PA}(\mathrm{r}=0.49$, $\mathrm{p}<0.0001$ for albumin; $\mathrm{r}=0.28, \mathrm{p}=0.031$ for creatinine) and inversely correlated with ECM/BCM ratio $(r=-0.42, p=0.001$ for albumin; $r=-$ $0.24, \mathrm{p}=0.07$ for creatinine). $\mathrm{nPCR}$ directly correlated with BMI ( $\mathrm{r}=$ $0.24, \mathrm{p}=0.08)$ and $\mathrm{PA}(\mathrm{r}=0.26, \mathrm{p}=0.049)$.

Patients were divided into 2 groups based on combined median values of BMI, ECM/BCM ratio and PA. Group 1: patients with BMI $\leq 24.72 \& \mathrm{PA} \leq 6.0 \& \mathrm{ECM} / \mathrm{BCM}$ ratio $\geq 1.18$. Group 2: patients with $\mathrm{BMI}>24.72 \& \mathrm{PA}>6.0$ \& $\mathrm{ECM} / \mathrm{BCM}$ ratio $<1.18$. As reflected by BIA indexes, group 2 patients had better nutritional status and were healthier than group 1 patients. Levels of nutritional markers such as albumin, creatinine and nPCR were significantly higher in Group 2 patients compared to those in Group 1 patients (Table 1).

\section{Body composition parameters and survival}

Patients were followed maximum up to 11 years. Three patients were followed up to 11 years. Fourteen patients were followed between 4 to 8 years. Twenty patients were followed between 2 to 4 years and number of patients followed less than 2 years was 26 . Mean and median follow-up were 2.98 and 2.31 years respectively. During the study period, 26 patients (42\%) expired. Patients who survived had higher BMI ( $27.61 \pm 7.38$ vs. $\left.23.68 \pm 4.2 \mathrm{Lbs} \mathrm{inch}^{2} \mathrm{p}=0.05\right)$, PA (6.54 \pm 1.72 vs. $5.17 \pm 1.30$ degree, $\mathrm{p}=0.017)$ and lower $\mathrm{ECM} / \mathrm{BCM}$ ratio ( $1.15 \pm 0.19$ vs. $1.31 \pm 0.18, \mathrm{p}=0.04)$ compared to those who expired.

We stratified each enrollment BIA parameter into 3 equal groups: BMI ( $\leq 23.15,23.18-26.63 \& \geq 26.85$ Lbs/inch $\left.{ }^{2}\right)$, PA $(<5.1,5.1-6.8 \&$

\begin{tabular}{|l|l|l|l|}
\hline Variable & Group 1 patients & Group 2 patients & p-value \\
\hline Albumin $(\mathrm{g} / \mathrm{dL})$ & $3.34 \pm 0.47$ & $4.09 \pm 0.56$ & $<0.0001$ \\
\hline Creatinine $(\mathrm{mg} / \mathrm{dL})$ & $9.41 \pm 3.21$ & $14.60 \pm 4.33$ & 0.001 \\
\hline $\mathrm{nPCR}(\mathrm{g} / \mathrm{kg} / \mathrm{day})$ & $0.805 \pm .23$ & $1.00 \pm 0.25$ & 0.034 \\
\hline
\end{tabular}

nPCR: normal protein catabolic rate

Group 1: Patients with $\mathrm{BMI} \leq 24.72, \mathrm{PA} \leq 6.0$ \& $\mathrm{ECM} / \mathrm{BCM}$ ratio $\geq 1.18$ Group 2: Patients with $\mathrm{BMI}>24.72, \mathrm{PA}>6.0$ \& $\mathrm{ECM} / \mathrm{BCM}$ ratio $<1.18$

Table 1: Biochemical nutritional markers by body composition in peritoneal dialysis patients.
$>6.80$ degrees $)$ and $\mathrm{ECM} / \mathrm{BCM}$ ratio $(<1.1,1.1-1.3 \&>1.30)$. On 11 years of observation, the cumulative observed survival of patients with enrollment $\mathrm{BMI} \geq 26.85 \mathrm{Lbs} / \mathrm{inch}^{2}$ was significantly better than that of patients with BMI $\leq 23.15$ ( $\mathrm{p}=0.02$ ) (Figure 1). In Cox's multivariate analysis adjusting for age, race, gender, diabetes, hypertension, history of heart disease and human immunodeficiency virus (HIV) seropositivity, BMI as a continuous variable was an independent predictor of mortality, although the results did not reach statistical significance (Relative risk: 0.87, $\mathrm{p}=0.09$ ).

PD patients with enrollment PA $>6.8^{\circ}$ had significantly better survival than those of patients with lower PA groups such as, 5.1 to $6.8^{\circ}(\mathrm{p}=0.014)$ and $<5.1^{\circ}(\mathrm{p}=0.001)$ respectively (Figure 2). In Cox's multivariate analysis adjusting for age, race, gender, diabetes, hypertension, history of heart disease and HIV seropositivity, PA was a significant independent predictor of mortality (Relative risk: 0.52 , $\mathrm{p}=0.009$ ). For each unit increase in PA, there was a $48 \%$ decrease in the relative risk of death.

Cumulative observed survival by $\mathrm{ECM} / \mathrm{BCM}$ ratio is shown in (Figure 3). Patients of the lowest ECM/BCM ratio tertile had significantly better survival compared to those of higher tertiles (ECM/ $\mathrm{BCM}$ ratio $<1.1$ vs $>1.3, \mathrm{p}=0.0026 ;<1.1$ vs.1.1-1.3, $\mathrm{p}=0.011 ; 1.1-1.3$ vs. $>1.3, \mathrm{p}=0.04$ ). In Cox's multivariate analysis adjusting for age, race, gender, diabetes, hypertension, history of heart disease and HIV seropositivity, ECM/BCM ratio was a significant independent predictor of mortality (Relative risk: 1.039, $\mathrm{p}=0.035$ ). For one per cent increase in $\mathrm{ECM} / \mathrm{BCM}$ ratio, there was a $3.9 \%$ decrease in the relative risk of death.

\section{Discussion}

We have shown that BIA derived body composition indexes are strongly associated with nutritional markers and are significant predictors of long term survival in PD patients. Our data indicate that albumin, a marker of visceral protein store and creatinine, a marker of somatic protein store are strongly associated with BIA derived body composition indexes, which is in agreement with our previously published reports $[12,14]$. We divided the patients into two groups based on combined median values of BMI, PA and ECM/ $\mathrm{BCM}$ ratio. Group 1 patients were more malnourished than group 2 patients. Serum albumin, creatinine and nPCR were significantly lower in Group 1 patients than those in Group 2 patients implicating the body composition parameters reflect nutritional status in PD patients. Among the BIA derived body composition parameters, PA may be the best index of nutritional status, as indicated by its strong association with all the three nutritional markers, albumin, creatinine and nPCR examined in this study. Significant correlation between PA and nutritional parameters in PD patients has been recently reported $[12,16]$. Measurement of PA has been reported to be a simple and reliable method for the routine assessment of nutritional status in continuous peritoneal dialysis patients [17].

The important finding of our study is that BMI, PA and ECM/BCM ratio, important markers of nutritional status, significantly predict long term survival in PD patients. Because of lack of recommendation for clinical thresholds of BIA parameters in PD patients, the patients were divided into tertiles based on the values for BMI, PA and ECM/ $\mathrm{BCM}$ ratio. This will add new information regarding the survival of patients based on two generated cut off points for the above variables. Our observation that higher enrollment BMI is significantly associated with increased survival (Figure 1) in PD patients is in agreement with previously published report by Johnson et al. who reported that obesity conferred a significant survival advantage in their PD patients [18] and 
by deMutsert et al that PD patients with a low BMI during dialysis have a twofold increased mortality risk [19]. However in Cox's multivariate model when we adjusted for the confounding variables, the association between BMI and survival did not reach statistical significance $(\mathrm{p}=0.09)$. The association between BMI and outcome of PD patients is controversial, but so-called obesity paradox (the higher BMI the longer survival) remains frequently reported. Aslam et al. reported that BMI above normal is not associated with any increased or decreased risk of death in patients on PD for 2 years [20]. In contrast, Zhou et al. have recently reported that normal BMI at the commencement of PD had significant survival advantage in their patient population [21]. However, McDonald et al. reported that obesity at the commencement of PD therapy is a significant risk factor for death and technique failure [22].

PA reflects cellular health and nutritional status in PD patients. In this study, PA was inversely associated with mortality risk and

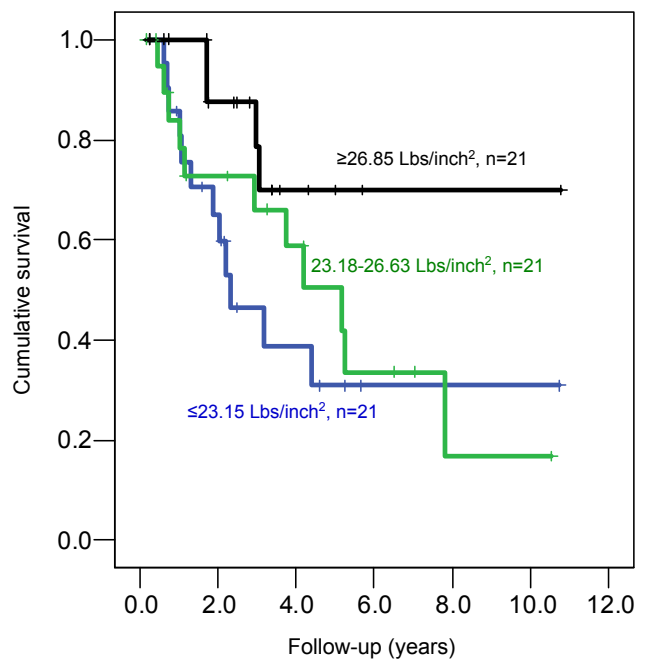

Figure 1: Observed patient survival by enrollment body mass index (BMI) in $\mathrm{PD}$ patients followed maximum up to 11 years. For $\geq 26.85$ vs. $\leq 23.15 \mathrm{Lbs} /$ inch $^{2}, p=0.02$.

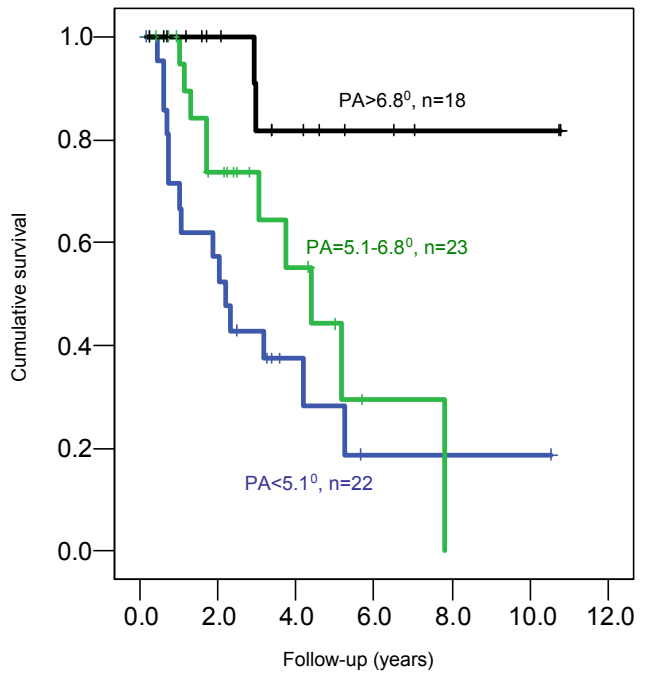

Figure 2: Observed patient survival by enrollment phase angle (PA) in PD patients followed maximum up to 11 years. $P A<5.1 \mathrm{vs}$. $>6.80, p=0.001 ; \mathrm{PA}<5.1$ vs. $5.1-6.80, p=0.04 ; 5.1-6.80$ vs. $>6.80 p=0.014$.

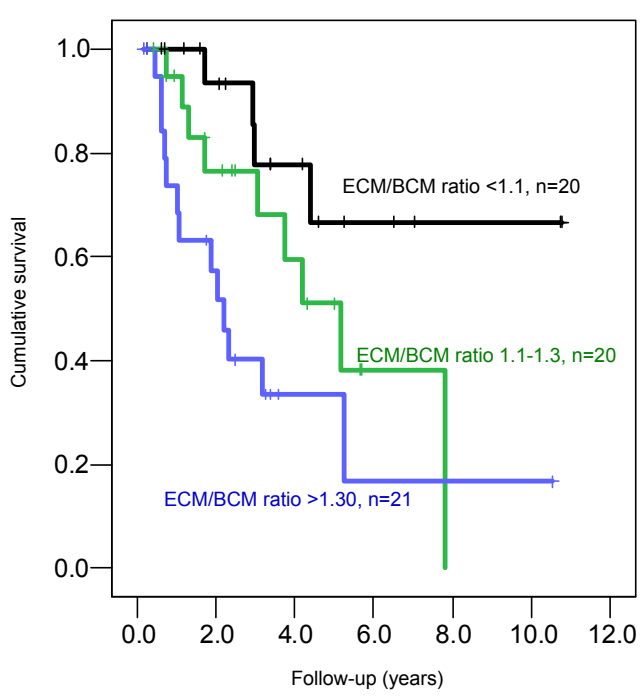

Figure 3: Observed patient survival by enrollment ECM/BCM ratio (ECM/BCM) in $\mathrm{PD}$ patients followed maximum up to 11 years. $E C M / B C M$ ratio $<1.1$ vs. $>1.3$, $p=0.0026 ;<1.1$ vs. $1.1-1.3, p=0.011 ; 1.1-1.3$ vs. $>1.3, p=0.04$

independently predicted 11 year's survival in PD patients. This confirms the previously published reports concerning the prognostic ability of PA in PD patients followed up to more than 5 years $[16,23]$. $\mathrm{ECM} / \mathrm{BCM}$ ratio is one of the most sensitive marker of malnutrition, higher the ECM/BCM ratio, poorer the nutritional status. In this study, higher values of ECM/BCM ratio were independently associated with increased mortality risk in $\mathrm{PD}$ patients followed up to 11 years which confirms our previous report with 8 years of follow-up.

Our study has several limitations. Although BIA measurements correlate well with gold standard methodology and BIA has been validated for dialysis patients, this method has some limitations including variability of BIA technique. The results of the BIA analysis may be influenced by hydration status, body position during procedure, recent physical activity and food consumption etc. Accuracy of the method can be improved by following the guidelines such as the use of same machine by the same operator, reducing the disturbances in fluid distribution, restricting food and alcohol consumption and exercise prior to the testing.

In summary, our study showed that enrollment BMI, PA and ECM/ $\mathrm{BCM}$ ratio which reflect nutritional status and are important predictors of survival in PD patients followed up to 11 years. However, the dietary and therapeutic intervention studies are needed to demonstrate if improvement in BIA parameters and nutritional status are associated with greater survival in $\mathrm{PD}$ patients.

\section{Acknowledgements}

The authors received no outside funding for this study. Part of this material was presented in poster form at the $44^{\text {th }}$ Annual meeting of the American Society of Nephrology, November 8-13, 2011.

\section{Disclosures}

The authors of this manuscript have no conflicts of interest to disclose.

\section{References}

1. U.S. Renal Data System (2011) USRDS 2011 Annual Data Report: Atlas of End-Stage Renal Disease in the United States, National Institute of Health, National Institute of Diabetes and Digestive and Kidney Diseases, Bethesda, MD.

2. Mehrotra R, Kopple JD (2003) Protein and energy nutrition among adult patients treated with chronic peritoneal dialysis. Adv Ren Replace Ther 10: 194-212. 
Citation: Avram MM, Fein PA, Agahiu S, Hartman W, Chattopadhyay J (2012) Body Composition, Nutritional Status and Mortality in Peritoneal Dialysis Patients. J Nephrol Therapeutic S10:003. doi:10.4172/2161-0959.S10-003

Page 4 of 4

3. McCusker FX, Teehan BP (1997) Peritoneal dialysis: an evolving understanding. Semin Nephrol 17: 226-238.

4. Wang AY (2011) Energy intake and energy expenditure profiles in peritonea dialysis patients. J Ren Nutr 21: 31-34

5. Jones CH, Newstead CG, Wills EJ, Davison AM (1997) Serum albumin and survival in CAPD patients: the implications of concentration trends over time. Nephrol Dial Transplant 12: 554-558.

6. Avram MM, Bonomini LV, Sreedhara R, Mittman N (1996) Predictive value of nutritional markers (albumin, creatinine, cholesterol, and hematocrit) for patients on dialysis for up to 30 years. Am J Kidney Dis 28: 910-917.

7. Avram MM, Goldwasser P, Erroa M, Fein PA (1994) Predictors of surviva in continuous ambulatory peritoneal dialysis patients: the importance of prealbumin and other nutritional and metabolic markers. Am J Kidney Dis 23 91-98.

8. Avram MM, Sreedhara R, Fein P, Oo KK, Chattopadhyay J, et al. (2001) Survival on hemodialysis and peritoneal dialysis over 12 years with emphasis on nutritional parameters. Am J Kidney Dis 37: S77-80.

9. Dong J, Li Y, Xu Y, Xu R (2011) Daily protein intake and survival in patients on peritoneal dialysis. Nephrol Dial Transplant 26: 3715-3721.

10. Leinig CE, Moraes T, Ribeiro S, Riella MC, Olandoski M, et al. (2011) Predictive value of malnutrition markers for mortality in peritoneal dialysis patients. J Ren Nutr 21: 176-183

11. Woodrow G, Devine Y, Cullen M, Lindley E (2007) Application of bioelectrical impedance to clinical assessment of body composition in peritoneal dialysis. Perit Dial Int 27: 496-502

12. Fein PA, Gundumalla G, Jorden A, Matza B, Chattopadhyay J, et al.(2002) Usefulness of bioelectrical impedance analysis in monitoring nutrition status and survival in peritoneal dialysis patients. Adv Perit Dial 18: 195-199.

13. Edefonti A, Picca M, Damiani B, Garavaglia R, Loi S, et al. (2001) Prevalence of malnutrition assessed by bioimpedance analysis and anthropometry in children on peritoneal dialysis. Perit Dial Int 21: 172-179.
14. Avram MM, Fein PA, Borawski C, Chattopadhyay J, Matza B (2010) Extracellular mass/body cell mass ratio is an independent predictor of survival in peritoneal dialysis patients. Kidney Int Suppl S37-40.

15. Kaplan EL, Meier P (1958) Nonparametric estimation from incomplete observations. J Am Stat Assoc 53: 457-481.

16. Abad S, Sotomayor G, Vega A, Pérez de José A, Verdalles U, et al. (2011) The phase angle of the electrical impedance is a predictor of long-term survival in dialysis patients. Nefrologia 31: 670-676.

17. Passadakis P, Sud K, Dutta A, Singhal M, Pettit J, et al. (1999) Bioelectrica impedance analysis in the evaluation of the nutritional status of continuous ambulatory peritoneal dialysis patients. Adv Perit Dial 15: 147-152.

18. Johnson DW, Herzig KA, Purdie DM, Chang W, Brown AM, et al. (2000) Is obesity a favorable prognostic factor in peritoneal dialysis patients? Perit Dia Int 20: 715-721.

19. de Mutsert R, Grootendorst DC, Boeschoten EW, Dekker FW, Krediet RT (2009) Is obesity associated with a survival advantage in patients starting peritoneal dialysis? Contrib Nephrol 163: 124-131.

20. Aslam N, Bernardini J, Fried L, Piraino B (2002) Large body mass index does not predict short-term survival in peritoneal dialysis patients. Perit Dial Int 22 191-196.

21. Zhou H, Cui L, Zhu G, Jiang Y, Gao X, et al. (2011) Survival advantage of normal weight in peritoneal dialysis patients. Ren Fail 33: 964-968.

22. McDonald SP, Collins JF, Johnson DW (2003) Obesity is associated with worse peritoneal dialysis outcomes in the Australia and New Zealand patien populations. J Am Soc Nephrol 14: 2894-2901.

23. Avram MM, Fein PA, Rafiq MA, Schloth T, Chattopadhyay J, et al. (2006) Malnutrition and inflammation as predictors of mortality in peritoneal dialysis patients. Kidney Int 70: S4-S7. 